\title{
Modeling Reach Motions Using Functional Regression Analysis
}

Julian J. Faraway University of Michigan 
The appearance of this ISSN code at the bottom of this page indicates SAE'S consent that copies of the paper may be made for personal or internal use of specific clients. This consent is given on the condition, however, that the copier pay a $\$ 7.00$ per article copy fee through the Copyright Clearance Center, Inc. Operations Center, 222 Rosewood Drive, Danvers, MA 01923 for copying beyond that permitted by Sections 107 or 108 of the U.S. Copyright Law. This consent does not extend to other kinds of copying such as copying for general distribution, for advertising or promotional purposes, for creating new collective works, or for resale.

SAE routinely stocks printed papers for a period of three years following date of publication. Direct your orders to SAE Customer Sales and Satisfaction Department.

Quantity reprint rates can be obtained from the Customer Sales and Satisfaction Department.

To request permission to reprint a technical paper or permission to use copyrighted SAE publications in other works, contact the SAE Publications Group.

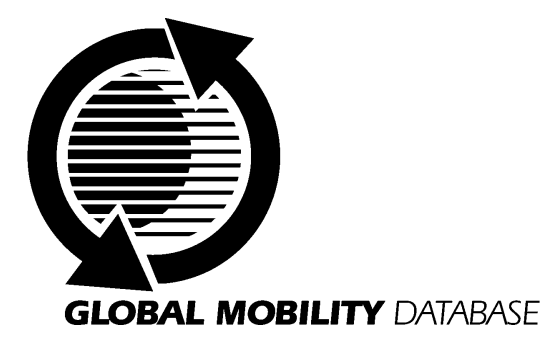

All SAE papers, standards, and selected books are abstracted and indexed in the Global Mobility Database

No part of this publication may be reproduced in any form, in an electronic retrieval system or otherwise, without the prior written permission of the publisher.

ISSN 0148-7191

Copyright $(2000$ Society of Automotive Engineers, Inc.

Positions and opinions advanced in this paper are those of the author(s) and not necessarily those of SAE. The author is solely responsible for the content of the paper. A process is available by which discussions will be printed with the paper if it is published in SAE Transactions. For permission to publish this paper in full or in part, contact the SAE Publications Group.

Persons wishing to submit papers to be considered for presentation or publication through SAE should send the manuscript or a 300 word abstract of a proposed manuscript to: Secretary, Engineering Meetings Board, SAE.

\section{Printed in USA}




\title{
Modeling Reach Motions Using Functional Regression Analysis
}

\author{
Julian J. Faraway \\ University of Michigan
}

Copyright @ 2000 Society of Automotive Engineers, Inc.

\begin{abstract}
This article describes a method for predicting human motion where some part of the body, such as the pelvis or foot does not move. The posture at any given time can be approximated using a linkage of articulated segments. The angles between the segments describe the posture. During the reach, these angles will vary describing a function that varies over time. Data may be collected on individuals reaching to a variety of targets. We describe a functional regression model for predicting the angles as they change with time as a function of the target being reached, the anthropometry and other characteristics of the individual. The model may be used to predict the motion of new individuals reaching to new targets not contained within the data. The model can also be used to describe the effects of factors such as age on reaching motions.
\end{abstract}

Although the main purpose of this article is to describe the methodology, we demonstrate its use on a large database of reaching motions collected by HuMoSim at the University of Michigan. We discuss the accuracy of the predictions and the difficulties ensuring that the hand reaches the intended target.

\section{INTRODUCTION}

The reaching motions of humans are dependent on many factors. Reach locations, seat type, anthropometry, age and gender, to name a few, are all factors that can affect dynamic reach postures. It is important to under-stand how reaches vary according to these factors when designing workplaces and vehicle interiors. One design approach is to build prototypes and test them using real human subjects. This approach is reliable but expensive and time consuming. Design is often an iterative process so several prototypes may need to be tested. An alternative approach is to construct a virtual prototype using CAD software. Virtual humans may be placed in the software and we may observe how they reach various locations ac-cording to their height, age, gender and other factors. In this paper I describe a statistical model for animating the reaches of these virtual humans.
The primary objective of this article is to describe the methodology, but we shall also illustrate the effectiveness of the tools we provide on some data. This data is described in the next section. The linkage can be described in terms of the angles formed by the segments of that link-age. In the following section, we show how to model these angles as they change over time using functional regression analysis. In the next section, we look at how well these models can be expected to fit and some difficulties with prediction.

\section{DATA}

In 1998, the Human Motion Simulation Laboratory (HUMOSIM) at the University of Michigan conducted a set of experiments concentrated on the motions of seated people performing right handed reaches to a spatially, well dispersed set of targets. The hand motion began from either in front of the person on a small table, or in the 2 o'clock position on a steering wheel, and proceeded to a specified target locations, paused for a few seconds, and returned to the initial position. The reaches to the target and the return reaches were modeled separately. A total of about 8000 motions were performed by a group of 20 subjects.

The subjects were selected to provide a means to assess the effects of anthropometry (height in particular), gender, and age on the motions. The subjects ranged from very short to very tall and from 20 to 60 years of age. Three different seating environments were examined: a car seat with medium side bolsters, a bus/ light truck seat, and an industrial seat with a narrow backrest. During the industrial seated reaches, motions were performed with and with-out a light hand load (which was set to load the shoulder at between 6\% to $15 \%$ of extended arm shoulder strengths).

Two different motion capture systems were simultaneously used to estimate joint angle changes throughout the motions: an optical reflective marker system (Qualysis MacReflex) and an electromagnetic (Ascension Flock-of-Birds). A seven-link kinematic model was created with joint centers estimated from the 
captured motion data. Joint angles were computed by processing the motion data through a version of JACK(tm) software configured to estimate 19 global and 11 local angles.

The first step of the data analysis is to extract the portions of the recorded motion where the subject was reaching. It is not easy to precisely define the beginning and end of a reach. The subjects are moving slightly even when they are at rest and some motion, in particular the head, sometimes occurs before the hand leaves its rest position and after it reaches the target. Nevertheless, a precise and consistent definition is required if we are to compare reaches across different subjects and targets. Our procedure for chopping the data is based on the finger position. We computed the distance of the finger from its rest position and used this to determine the beginning and end of the reach. There is a pause when the target is reached, the duration of which is random and may be different at different times. We have no particular interest in this so we removed these periods also. We divide the motion into reaching and returning parts.

We emphasize that the methodology presented here could be applied to data collected in different ways for different linkages. We have provided some details of the data collection to allow the reader to follow the examples below but the data and the conclusions drawn from this data are not the focus of this article.

\section{FUNCTIONAL REGRESSION MODEL}

After extracting the data, for any selected angle and specified motion, we have a sequence of angles from the start to the end. These sequences are of different lengths because some targets are further away than others and people reach at different speeds. Plots of the right elbow included angle of 20 subjects reaching to a location somewhat to the right and front and about the same height as the initial position of the hand are shown in Figure 1. We need to standardize the lengths of the curves if we are to compare different reaches. We define $t=0$ to be the start of the motion and $t=1$ to be the end of the motion. We record and save the sequence length as the actual time that the motion took. We can analyze these actual times independently. For now we concentrate on the shape of the motion without regard to the actual amount of time taken. We can put the time scaling back in later. We show the same curves rescaled to a common length in Figure 2.

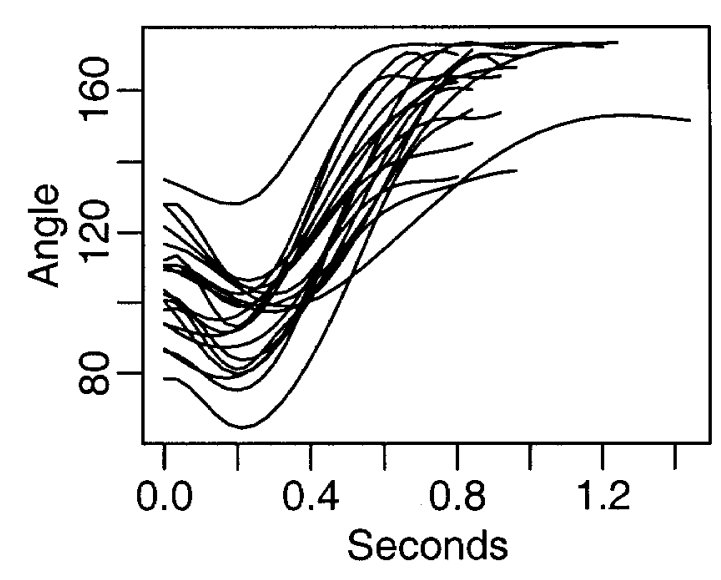

Figure 1. Right elbow included angle for 20 subjects reaching to the right and forward of the initial hand position

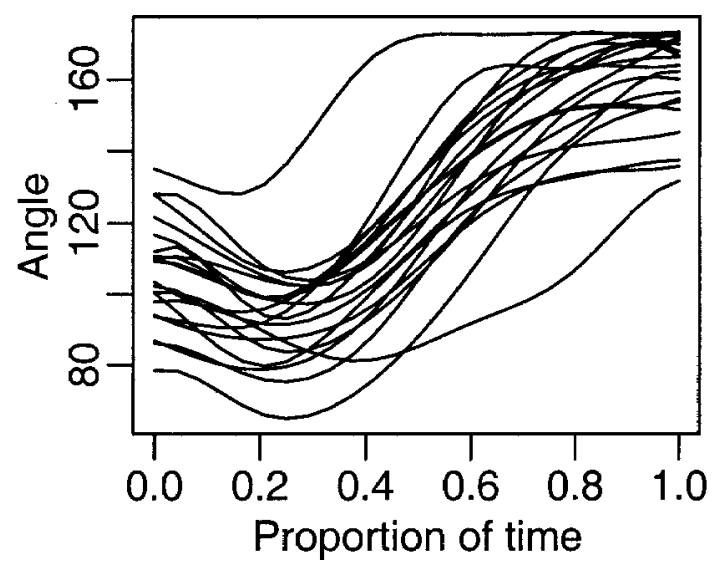

Figure 2. The same angle curves as in Figure 1 but scaled so that the horizontal axis is the proportion of time taken for the reach

Suppose the rescaled angle functions are given by $y(t)=$ $\left(y_{1}(t), \ldots y_{n}(t)\right)$. These angle functions might be expected to depend on certain covariates such as the location of the target being reached, the age and anthropometry of the subject and other factors. For the $i^{\text {th }}$ subject, we collect these predictors in a vector $x_{i}$. Typically, the first term in this vector is one. We then propose a functional linear model for the angle functions:

$$
y_{i}(t)=x_{i}^{T} \beta(t)+\epsilon_{i}(t)
$$


Notice that this is comparable to a standard regression model but the response is now a function as is the error term $\epsilon_{\mathrm{i}}(t)$. The regression coefficients $\beta(t)$ are now a vector of functions. A general introduction to the area of functional data analysis may be found in [5]. The particular coefficient function for a given covariate will now represent the effect on the response of that covariate over the duration of the reach. We can now estimate $\beta(t)$ using the method of least squares applied across the whole time period. The estimator takes a familiar form:

$$
\hat{\beta}(t)=\left(X^{T} X\right)^{-1} X^{T} \mathbf{y}(t)
$$

where $X$ is the matrix whose rows are given by the $X_{i}$ 's.

Although this is conceptually illuminating, it is not practical since we cannot observe a $y_{\mathrm{i}}(t)$ at all possible $t$. One approach is to approximate the functions on a grid of values. This was done in [2] with further developments in [6]. The major drawback is that a fine grid of values is necessary for accurate representation and so large matrices of values are required. These can be burdensome to manipulate and store.

For the current data, we have taken a basis function approach. We use standard cubic B-splines. We represent the angle curves as linear combinations of these basis functions, $\phi_{j}(t)$. An angle curve $y_{\mathrm{i}}(t)$ is represented as

$$
y_{i}(t)=\sum_{i=1}^{m} y_{i j} \psi_{j}(t)+\epsilon_{(}(t)
$$

where the coefficients $y_{\mathrm{ij}}$ are found by minimizing

$$
\int_{0}^{1}\left(y_{i}(t)-\sum_{j=1}^{m} y_{i j} \psi_{j}(t)\right)^{2} d t
$$

We observe $y(t)$ at some set of time points and so the coefficients $y_{i j}$ can be estimated using least squares. It is not uncommon for some parts of the curve to be missing due to data collection problems. This method can tolerate a certain amount of missing data. Furthermore, if measurement or processing systems are prone to produc-ing outliers, a robust fitting method may be substituted for least squares. Given that human motion is usually quite smooth, it is not necessary to have a large number of basis functions. In this particular application, we found that eight basis functions were adequate. Note that eight grid points would not have been adequate for the approach mentioned above so this allows for a large reduction in the data needed for fitting these models and in the representation of the predictive models.

Using more than eight basis functions did not improve the fit significantly and, furthermore, restricting the number of coefficients has the advantage of smoothing out any small irregularities due to measurement error. We chose cubic B-splines for the basis because of their well-known stability for numerical calculations in contrast to polynomials. The cubic degree also allows for continuous first and second derivatives which is important if velocity and acceleration are needed.

Thus we can write the model in the approximate form

$$
Y_{n \times m} \psi_{m \times 1}(t)=X_{n \times p} B_{p \times m} \psi_{m \times 1}(t)+\epsilon_{n \times 1}(t)
$$

or factoring out the $\psi(t)$, we can write it in the simpler form:

$$
Y_{n \times m}=X_{n \times p} B_{p \times m}+\epsilon_{n \times m}
$$

which is now a multivariate multiple regression model where the coefficient matrix $B$ may be simply estimated using least squares. We may estimate $B$ by least squares as in

$$
\hat{B}=\left(X^{T} X\right)^{-1} X^{T} Y
$$

We may use the standard methods of statistical inference such as testing using this model. Details of such methods may be found in texts such as [4]. For prediction and interpretation purposes, it is necessary to transform back from this basis function representation to the original form. For example, for a particular $\hat{\beta}(t)$ we would need to take a linear combination of cubic splines represented by the appropriate row of $\hat{B}$. Explicitly, we may recover the original coefficient functions by

$$
\hat{\beta}_{p \times 1}(t)=\hat{B}_{p \times m} \psi_{m \times 1}(t)
$$

and predict future responses given a predictor value $x_{0}$ by

$$
\hat{y}_{0}(t)=x_{01 \times p} \hat{B}_{p \times m} \psi_{m \times 1}(t)
$$

We estimate the variance of the prediction by

$$
v \hat{a} r\left(\hat{y}_{0}(t)\right)=\psi^{T}(t) \hat{\Sigma} \psi(t) x_{0}^{T}\left(X^{T} X\right)^{-1} x_{0}
$$

where

$$
\hat{\Sigma}=\frac{\hat{\epsilon}^{T} \hat{\epsilon}}{n-p} \quad, \quad \hat{\epsilon}=Y-X \hat{B}
$$

The pointwise variance of the coefficient functions may be obtained from

$$
v \hat{a} r \hat{\beta}_{i}(t)=\psi^{T}(t) \hat{\Sigma} \dot{\psi}(t)\left(X^{T} X\right)_{i i}^{-1}
$$

We may also compute the $R^{2}$ statistic and the F-statistic at each point in time using the standard regression formulae.

We must decide which predictors to include in the model. How we make that choice depends somewhat on our 
objectives. If the main purpose of the investigation are scientific questions concerning the effect of various predictors such as age on motion, then we can use the inferential tools concerning the statistical significance of the predictors. For example, Figure 3, shows the effect of stature over time on the right elbow included angle. The model was restricted to 16 targets on the left side of the body when reached with the right hand. Predictors included were the target coordinates, the stature, the age, the gender and the time taken to complete the reach. We can see that the effect of stature varies across the duration of the reach. We see that taller persons do not open their elbows as much as shorter people earlier in the reach, but there is little difference towards the end of the reach. We can see that the magnitude of the effect could be as much as about 4 degrees for each $10 \mathrm{~cm}$ difference in height. We see that the effect is statistically significant during the initial part of the motion because zero is not included in the interval. Towards the end of the reach, there is no statistically significant difference between taller and shorter persons. The $95 \%$ pointwise confidence bands are useful for judging the statistical significance of the effect. Simultaneous confidence intervals are also useful — these are described in [7]. Of course, there is much interest in discerning the effect of stature, age and sex and other factors on motion. For more on this, see [1].

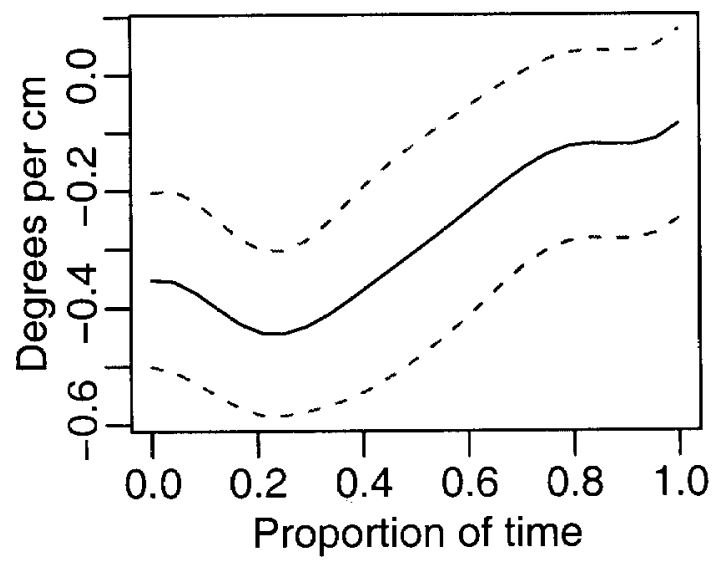

Figure 3. The solid line shows effect of stature on the right elbow included angle per $\mathrm{cm}$ of height over time. $95 \%$ pointwise confidence bands are shown as dashed lines

If we are more interested in prediction, then predictive accuracy becomes the main concern. For the industrial data mentioned above, we built a model that included age, gender, load, stature, 3D coordinates of the target. We used certain interactions of these terms. We found no significant improvement in fit by including other anthropometric terms. This is because such measures are usually strongly correlated with stature and so there is no significant advantage in including such terms in the model. We describe the accuracy of this model in the next section.

\section{MODEL FIT}

No model is perfect - we can only aim for an accept-able approximation to reality. We may predict the angles for the motion of a given individual, but we might expect that when that person actually makes the reach, the reality will be somewhat different. How much error can be expected for our predictions? Remember that the same person will vary in their motions to the same location and that persons with the same anthropometry, age and gender will not reach in exactly the same way. This kind of variation cannot be overcome, so there is a lower bound on the error below which we could never expect to go. We designed the experiments to have replicates for certain targets so that we could study this variability. Each subject was asked to reach 10 prechosen targets twice in the industrial setting. These replicates help us to understand the natural variation existing in human motion. We identify two types of variation. A single individual will vary in his motions - this is termed variation within a subject. Different individuals will vary in their motions, even after we adjust for significant factors, such as stature. This is termed variation between subjects. Table 1 the standard deviations in the industrial experiment along with a measure of the predictive precision of our particular model:

Table 1. Estimated standard deviation for between subject and within subject variability for selected angles averaged across subjects and time. Residual standard error for the functional regression model is also shown

\begin{tabular}{lrrr} 
Angle & Between & Within & Model \\
\hline trunk flexion & 6.32 & 2.13 & 5.45 \\
trunk lateral & 4.38 & 2.06 & 4.93 \\
trunk axial & 9.88 & 5.39 & 9.22 \\
upper arm horizontal & 19.64 & 8.65 & 20.08 \\
upper arm vertical & 7.84 & 4.39 & 8.58 \\
forearm horizontal & 11.13 & 6.35 & 12.84 \\
forearm vertical & 7.86 & 3.28 & 9.20
\end{tabular}

The results from this analysis reveal that within-individual variation is relatively smaller as might be expected. The between-individual variation is an estimate of the lower bound on how well any prediction model might be expected to perform when representing a well defined group of people. Notice that the model variation is not far from this estimated lower bound - in some cases it even goes below the bound. Remember that the between-individual variation is only an estimate. It uses only the ten targets at which replicates were made and we may not be completely removing the effects of age, stature and gender in computing this variation. Nevertheless, we can feel confident that our model cannot be significantly improved up-on beyond the between-individual variation values. The results for the car and truck data are qualitatively similar. 
If prediction is the final objective, the angles predicted by the model must be used to construct a dynamic linkage representing the motion. When this is done, the position of the hand at the end of the motion may not match with the specified target. In the example above, the discrepancy was around $5 \mathrm{~cm}$ on average. There are several reasons for this. Variability in the predicted or specified link lengths will cause some error. Even though the model appears to approach the upper limit in effectiveness as demonstrated in Table 1, there will still be some variation. However, there is a more fundamental reason for the error. The final hand position is a nonlinear function of the angles. This means that even if we considered data for a subject reaching to the same target, but using different postures and then averaged the angles for those postures, the predicted hand position using those averaged angles would likely be different from that seen in all the reaches. This means that any method that seeks to predict angles individually and then uses them to make predictions will suffer this problem.

One solution would be to impose a constraint on the estimation of the coefficients in the functional regression models for the angles that ensures that the hand hits the target. Such a constraint would also need to use the link lengths. This is very complicated and appears inconceivable for all except very simple linkages. An alternative is to correct the predicted linkage so that it does meet the endpoint constraints. One solution is described in [3].

\section{CONCLUSION}

The functional regression method is effective in predicting the angle profiles that describe a reaching motion. Successful implementation requires that a suitable linkage be specified. There is a great amount of choice in defining the angles describing this linkage. In particular, one must choose between global and local definitions of angles. For the example, the right elbow included angle is locally defined by the upper and lower arms whereas the angle of elevation of the lower arm might be defined with respect to a global coordinate system. If the functional regression model is to be used for explanatory purposes, it is usually better to use local angles since these are easy to interpret. However, if the model is to be used for predictive purposes, the choice of angles will be driven by the nature of the digital human software being used.
Functional regression methods exist for modeling a curve in terms of another curve and perhaps other variables see [5] or [7]. Such models would be useful for investigating, say, the relationship between shoulder and elbow angles. They are typically not useful for predicting motion using static inputs of the type described above since all the angles must be simultaneously predicted we do not have an angle curve available to help predict the others.

Functional regression models can also be used to predict the trajectories of body markers such as the hand. Work on this is in progress.

\section{ACKNOWLEDGMENTS}

Thank you to Don Chaffin and the other members of the HUMOSIM laboratory - Chuck Woolley, Jim Foulke and Woojin Park. Yao Wang assisted in the computations. The work was made possible by the funding of Ford, General Motors, Navistar, Johnson Controls and TRW.

\section{REFERENCES}

1. D. Chaffin, J. Faraway, X. Zhang, and C. Woolley. Stature, age, and gender effects on reach motion postures. Human Factors, 2000.

2. J. Faraway. Regression analysis for a functional response. Technometrics, 39:254-261, 1997.

3. J. Faraway, X. Zhang, and D. Chaffin. Rectifying postures reconstructed from joint angles to meet constraints. Journal of Biomechanics, 32:733-736, 1999.

4. R. Johnson and D. Wichern. Applied Mulivariate Statistical Analysis. Prentice Hall, New Jersey, 3rd edition, 1992.

5. J. Ramsay and B. Silverman. Functional Data Analysis. Springer, New York, 1997.

6. Qing Shen. Linear Models for a Functional Response. PhD thesis, Department of Statistics, University of Michigan, 1998.

7. Yao Wang. Some Issues in Functional Data Analysis: Basis Approach, Roughness, Mode and Modelling. $\mathrm{PhD}$ thesis, Department of Statistics, University of Michigan, 2000. 